\title{
FLUCTUATIONS OF CHERN-SIMONS NUMBERS IN THE SU(2) HIGGS MODEL
}

\author{
F. KARSCH, M.L.LAURSEN \\ HLRZ, c/o KFA Jülich, D-52425 Jülich, Germany \\ T. NEUHAUS and B. PLACHE \\ Fak. f. Physik, Univ. Bielefeld, P.O.Box 100191, D-39501 Bielefeld, Germany
}

\begin{abstract}
We investigate vacuum transitions in lattice higgs models at finite temperature. The 2 dimensional $U(1)$ Higgs model is used as a toy model. In the 4 dimensional $S U(2)$ Higgs model the region of the phase transition and temperatures above it are considered. The couplings $(\beta, \kappa, \lambda)=(2.25,0.27,0.5)$ and $(8.0,0.12996,0.0017235)$ correspond to masses in lattice units $\left(a_{\sigma} m_{H}, a_{\sigma} m_{W}\right)$ of $(0.02,0.05)$ and $(0.2,0.2)$, respectively. The algorithm is described and a parallelized version is proposed. Taking the influence of the finite lattice into account we discuss temperature effects. We compare our results with perturbative estimates and claim that they link low and high temperature approximations.
\end{abstract}

Keywords: Higgs model; vacuum structure; finite temperature; topology.

\section{Introduction}

Any theory to be considered seriously as a theory of the early universe must be able to predict its future behaviour from some given initial conditions. In case of the standard big bang model a hot and dense universe of temperature $100 \mathrm{MeV}$ is assumed to have existed at an early time. Applying general relativity and elementary particle interactions lead to an expanding universe with a microwave background radiation and certain proportions of nuclei, which may be found even today in the outer layers of old stars.

Nevertheless there are observations which do not come out of the model quite naturally, but have to be given as special input parameters. The flatness of the universe or its large scale structure are examples which might be solved by the concept of inflationary cosmology. ${ }^{1}$ This is achieved by extrapolating the theories of today (in the range of $10^{2} \mathrm{GeV}$ ) up to energies of approximately $10^{19} \mathrm{GeV}$.

In this context there also appear baryon number violating processes, thus prohibiting the possibility of a fixed baryon number of the universe since its beginning. ${ }^{2}$ In an attempt to explain the observed baryon number by elementary particle processes it is most natural to investigate the latest event in history which might account for it. Assuming the electroweak standard model to be valid up to energies 
of $10^{3} \mathrm{GeV}$, sufficient conditions to generate a baryon asymmetry are fulfilled: The anomal current allows baryon nonconservation, ${ }^{3}$ the electroweak phase transition at approximately $10^{2} \mathrm{GeV}$ ensures thermal nonequilibrium, and the KobayashiMaskawa mixing gives the required $C P$ violation. ${ }^{4}$ Therefore the standard electroweak model near and above its phase transition will be considered.

The following scenario may give some idea of the generation of a baryon number asymmetry: ${ }^{5}$ Approaching the electroweak phase transition from above the system stays in a metastable symmetric phase even slightly below the transition temperature. Small fluctuations of the cold, broken phase are suppressed due to the surface tension. At some temperature the fluctuations get big enough, and the transition into the broken phase proceeds via bubble formation. While in the symmetric phase baryon violation is still possible, any baryon number present in the broken phase will be conserved. The asymmetric reflection behaviour of baryons and antibaryons at the expanding bubble wall due to CP violation - antibaryons are easier reflected while baryons may pass through - leads to an accumulation of baryons inside the bubbles, while the surplus of antibaryons outside the bubbles disapears due to baryon number violation. When the whole universe is in the broken phase thus the observed baryon asymmetry of the universe is generated.

In the next section the model used for this investigation is introduced. The terms needed to describe baryon number violaton are developed in the third section. Some remarks about the algorithm follow in section four. Section five contains the results, and finally we give some perspectives.

\section{The Model}

Our approximation of the standard electroweak model by the pure $S U(2)$ Higgs model is justified because the $U(1)$ fields couple only weakly, and fermions can be neclected if the top quark is not too heavy. Then the Lagrangian of the system

$$
L=-\frac{1}{4} F_{\mu \nu}^{a} F^{\mu \nu a}+\left(D_{\mu} \Phi\right)^{\dagger}\left(D^{\mu} \Phi\right)+\lambda\left(\Phi^{\dagger} \Phi-v^{2} / 2\right)^{2}
$$

leads in the euclidean formulation on the lattice to the local action

$$
\begin{gathered}
S_{n}=-\frac{\beta \gamma_{g}}{2} \sum_{\nu=1,2,3} \operatorname{tr}\left(U_{n, 0 \nu}\right)-\frac{\beta}{2 \gamma_{g}} \sum_{1 \leq \mu<\nu} \operatorname{tr}\left(U_{n, \mu \nu}\right) \\
-\kappa \gamma_{h} \operatorname{tr}\left(\phi_{n}^{\dagger} U_{n, 0} \phi_{n+0}\right)-\frac{\kappa}{\gamma_{h}} \sum_{\mu=1,2,3} \operatorname{tr}\left(\phi_{n}^{\dagger} U_{n, \mu} \phi_{n+\mu}\right) \\
+\lambda\left(\phi_{n}^{\dagger} \phi_{n}-1\right)^{2}+\operatorname{tr}\left(\Phi^{\dagger} \Phi\right) .
\end{gathered}
$$

Here we use an anisotropic lattice with spacing $a_{\sigma(\tau)}$ and $N_{\sigma(\tau)}$ points in space (time) direction. The lattice anisotropy $\xi=a_{\sigma} / a_{\tau}$ is controled by the coupling anisotropies $\gamma_{g}$ for the gauge coupling $\beta=2 N / g^{2}$, and $\gamma_{h}$ for the hopping parameter $\kappa$. To leading order in the weak coupling expansion these anisotropies are equal to the lattice anisotropy $\xi$, but in a more precise investigation the lattice anisotropy must be calibrated by correlation functions. ${ }^{6}$ 
The temperature is introduced into quantum field theories by imposing periodic boundary conditions in time direction, its extension beeing the inverse temperature. Then on the lattice we have

$$
a_{\tau} N_{\tau}=\frac{1}{T}
$$

Usually physical quantities like correlation functions or masses are given in units of the spatial lattice spacing, $a_{\sigma}$. Then the temperature is

$$
a_{\sigma} T=\frac{a_{\sigma}}{a_{\tau} N_{\tau}}=\frac{\xi}{N_{\tau}}
$$

At some stages in this article it will be helpful to use the $2 \mathrm{~d}$ abelian Higgs model. Due to its lower dimensionality in configuration and internal space it is much more easy to simulate, while the special properties conserning baryon number violation are still present.

The model is defined analog to the $4 \mathrm{~d} S U(2)$ model; the gauge and Higgs fields

$$
\begin{aligned}
& A_{n, \mu}=e^{i \theta_{n, \mu}} \\
& \Phi_{n,}=R_{n} e^{i \varphi_{n}}
\end{aligned}
$$

become complex numbers, and the action at each lattice site $n$ is

$$
\begin{aligned}
S_{n} & =\frac{1}{a^{2} g^{2}}\left(1-\cos \left(\theta_{n, \square}\right)\right)+\lambda a^{2}\left(R_{n}^{2}-v^{2} / 2\right)^{2} \\
& +\sum_{\mu=1,2}\left(R_{n}^{2}+R_{n+\hat{\mu}}^{2}-2 R_{n} R_{n+\hat{\mu}} \cos \left(\varphi_{n}+\theta_{n, \mu}-\varphi_{n+\hat{\mu}}\right)\right)
\end{aligned}
$$

\section{Topological Properties}

There is a one to one correspondence between baryon number violating processes and vacuum transitions. The $S U(2)$ Higgs model has an infinite degenerate vacuum, which can be visualized by a periodic potential. ${ }^{7}$ The baryon number violation, which takes place when the system moves from one vacuum to another, can be expressed in terms of the gauge fields. This is possible, because the integral over the anomalous current

$$
\partial_{\mu} j_{B}^{\mu}=-\frac{n_{f}}{16 \pi^{2}} \operatorname{tr}\left(F_{\mu \nu} \tilde{F}^{\mu \nu}\right)
$$

gives the baryon number difference. ${ }^{3}$ Here $n_{f}$ is the number of fermion families. Assuming the system to be at times $t_{1,2}$ in a vacuum, the baryon number difference between these two vacua is given by

$$
\Delta B=-\frac{n_{f}}{16 \pi^{2}} \int_{t_{1}}^{t_{2}} d t \int d^{3} x \operatorname{tr}\left(F_{\mu \nu} \tilde{F}^{\mu \nu}\right)=-n_{f} Q,
$$

where $Q$ is called the topological charge. 
Semiclassicaly the transition between different vacua is given by the instanton, a field configuration interpolating between two vacua. The transition rate obtained by this method is tiny, and baryon number violation via this process is not expected to be measurable. ${ }^{3}$

There is another aproach to vacuum transitions. In the above mentioned potential picture a field configutration in the middle between two vacua on top of the potential barrier can be constructed for a fixed time. ${ }^{8}$ It is called sphaleron and has an energy of $\sim 10 \mathrm{TeV}$.

For the 2d abelian Higgs model an instanton configuration with a sphaleron inbetween with $n_{c s}=0.5$ can be constructed and visualized easily (fig. 1 ).

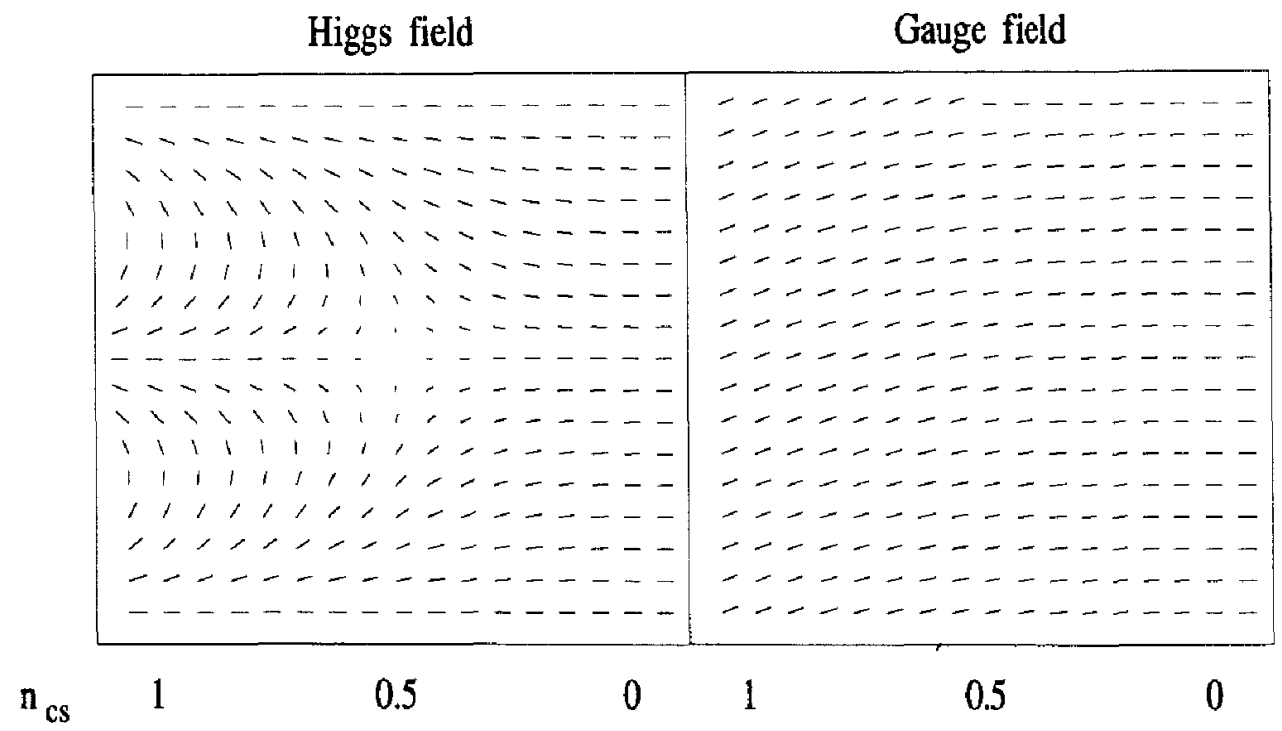

Fig. 1. Instanton configuration for the $2 \mathrm{~d}$ abelian model. Gauge fields in $A_{0}=0$ gauge are at the left, Higgs field radial part and argument are at the right side. The different vacua are on the left hand side $n_{c s}=1$ and on the right hand side $n_{c s}=0$ for both fields.

Heating the system to a temperature of the order of the sphaleron energy would result in a high probability to end in a different vacuum after cooling. From this picture two temperature regions with different transition behaviour are found. If the temperature is below the vector boson mass, the transition rate $\Gamma$ per unit time and volume is given by ${ }^{9}$

$$
\Gamma=0.007\left(\alpha_{w} T\right)^{4}\left(\frac{3 m_{W}}{T \alpha_{w}}\right)^{7} e^{-3 m_{W} / T \alpha_{w}}
$$

For high temperatures one may assume $m_{W} \sim T$, resulting in

$$
\Gamma \sim T^{4}
$$

with a proportionality constant between 0.1 and $1 .^{10}$

Investigating the behaviour of the $S U(2)$ Higgs system requires a classification of the field configurations at a fixed time in order to determine if the system is in a potential well or on top of the potential barrier. 
Due to its special form, the anomalous current (eq. 3.1) can be written as a divergence

$$
\partial_{\mu} K^{\mu}=-\frac{1}{16 \pi^{2}} \operatorname{tr}\left(F_{\mu \nu} \tilde{F}^{\mu \nu}\right)
$$

Then it is possible to write the baryon number difference in terms of the zeroth component of $K$ as the difference between two integrals:

$$
\Delta B=-n_{f}\left(\int d^{3} x K^{0}\left(t_{2}, x\right)-\int d^{3} x K^{0}\left(t_{1}, x\right)\right)=-n_{f}\left(n_{c s}\left(t_{2}\right)-n_{c s}\left(t_{1}\right)\right)
$$

The hereby defined Chern-Simons number $n_{c s}$ is integer for vacuum configurations and has half integer values for configurations on top of the potantial barrier, for example the sphaleron. Under a gauge transformations the number only changes by an integer, resulting in an invariant difference between Chern-Simons numbers, and thus baryon number.

\section{The Algorithm}

The definition of the Chern-Simons current $K_{\mu}$ (eq. 3.5) allows us to write the Chern-Simons number in terms of the gauge fields. Inserting

$$
K_{\mu}=-\frac{1}{8 \pi^{2}} \varepsilon_{\mu \nu \rho \sigma} \operatorname{tr}\left(A_{\nu}\left(\partial_{\rho} A_{\sigma}+\frac{2}{3} A_{\rho} A_{\sigma}\right)\right)
$$

into the definition (eq. 3.6) makes it possible to evaluate the Chern-Simons number for a given field configuration.

Our investigation consists in a generation of statistically independent field configurations by the Monte Carlo method. Measuring distributions of Chern-Simons numbers gives the effective Chern-Simons potential $V\left(n_{c s}\right)$, which is defined by

$$
\begin{aligned}
& P\left(n_{c s}\right)=\exp \left(-V\left(n_{c s}\right)\right)= \\
& \int d A d \Phi e^{-S(A, \Phi)} \delta\left(n_{c s}+\frac{1}{8 \pi^{2}} \int d^{3} x \varepsilon_{i j k} \operatorname{tr}\left(A_{i}\left(\partial_{j} A_{k}+\frac{2}{3} A_{j} A_{k}\right)\right)\right) .
\end{aligned}
$$

In a lattice formulation care has to be taken to preserve the transformation properties of the Chern-Simons number. Especially the change by an integer under gauge transformations cannot be achieved by a naive lattice definition of eq. (4.1). Seiberg's definition is well suited for our purpose. ${ }^{11}$

It requires the construction of an interpolating function in each 4 cube of the lattice; it depends only on the link matrices on the edges of each 4 cube. This function has to be integrated over and summed up for every cube.

But there some problems arise. Only one of the required three dimensional integrations can be performed analytically. As all the integrations are independent, they can all be done in parallel. This would be very simple if we could use one set of integration points for all the cubes. But usually for $90 \%$ of the cubes the interpolating function is smooth, thus requiring only a small number of integration 
points. The few remaining cubes have to be integrated on a finer grid, and the whole procedure is not straightforwardly parallelizable.

We propose the following procedure: First each cube is integrated with $P \times P$ and with $2 P \times 2 P$ integration points. If the difference between these integrations is less then some threshold $(0.01 \%)$, the integrations are assumed to be correct. Otherwise the integrations are repeated with a higher number of integration points. This procedure becomes inefficient when there is only a small number of cubes left. These are dealt with in a different way. The different integration points (usually of the order $64 \times 64$ up to $512 \times 512$ ) are now located on different sites and the function is now evaluated in parallel for one cube at a time.

The special values for an optimal program are coupling parameter and computer dependent. An initial value of $P=8$ and distribution of the last 8 to 16 cubes over the lattice proved to be a good choice for our task.

The calculations were done on the CRAY-YMP at HLRZ Jülich and on the NECSX3 of the University of Köln. In table 1 some average times for the evaluation of Chern-Simons numbers are given.

Table 1. Time needed to evaluate a single Chern-Simons number on the CRAY-YMP at couplings $\beta=2.25, \kappa=0.25,0.3,0.4$ and $\lambda=0.5$ as well as on the NEC-SX3 at $\beta=8, \kappa=0.12996$ and $\lambda=0.0017235$.

\begin{tabular}{|c|c|c|c|}
\hline \multicolumn{4}{|c|}{ CRAY-YMP } \\
\hline$N_{\sigma}$ & $N_{\tau}$ & $\kappa$ & $\bar{t} / N_{\sigma}^{3}[\mathrm{~ms}]$ \\
\hline 6 & 6 & 0.25 & 11.4 \\
6 & 6 & 0.30 & 10.0 \\
6 & 6 & 0.40 & 9.9 \\
\hline 8 & 8 & 0.30 & 9.2 \\
8 & 8 & 0.40 & 8.9 \\
8 & 4 & 0.30 & 9.5 \\
8 & 2 & 0.30 & 4.4 \\
\hline
\end{tabular}

\begin{tabular}{|c|c|c|c|}
\hline$N_{\sigma}$ & $N_{\tau}$ & $\xi$ & $\bar{t} / N_{\sigma}^{3}[\mathrm{~ms}]$ \\
\hline 4 & 4 & 1.0 & 45.2 \\
4 & 2 & 1.0 & 29.5 \\
4 & 2 & 2.0 & 11.2 \\
\hline 6 & 6 & 1.0 & 75.7 \\
6 & 2 & 1.0 & 27.2 \\
6 & 2 & 2.0 & 8.6 \\
\hline 8 & 8 & 1.0 & 76.2 \\
8 & 2 & 1.0 & 31.1 \\
8 & 2 & 2.0 & 10.2 \\
\hline
\end{tabular}

\section{Results}

The first part of the investigaton consisted of a measurement of the topological susceptibility of the $S U(2)$ Higgs system. By measuring the topological charge $Q$ of a field configuration it is possible to identify vacuum transitions. The topological susceptibility is related to the transition rate via ${ }^{10}$

$$
\Gamma=\left\langle Q^{2}\right\rangle /\left(2 N_{\sigma}^{3} N_{\tau}\right)
$$

These results are for $\beta=2.25$ and $\lambda=0.5$ on both sides of the phase transition at $\kappa=0.26065$, which corresponds to Higgs and vector boson masses of 0.02 and 0.05 in units of the lattice spacing. ${ }^{12}$

There is obviously a suppression of configurations with topological charges in the Higgs phase of the system (fig. 2) due to the nonvanishing Higgs expectation 


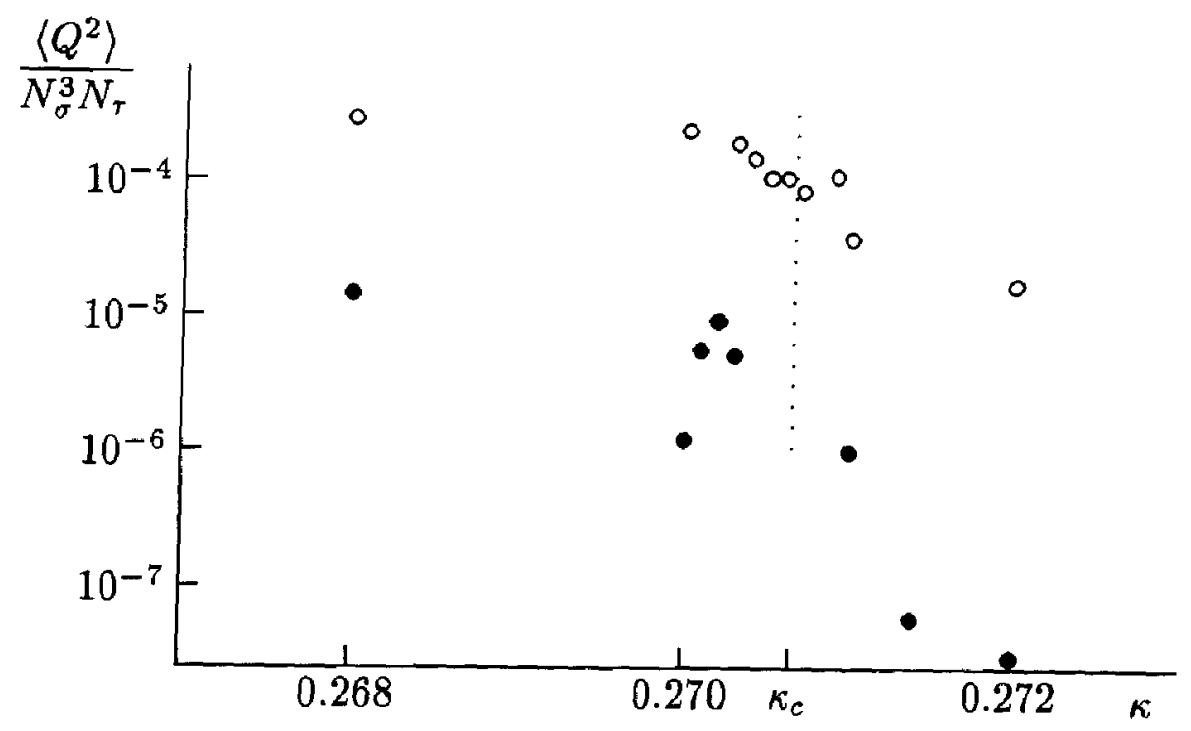

Fig. 2. Topological susceptibility at zero $(\circ)$ and finite $(\bullet)$ temperature.

value there. ${ }^{13}$ At higher temperature there is an additional suppression. Clearly instanton effects do not contribute to vacuum transitions.

Therefore only Chern-Simons numbers will be considered. Since the lattice is finite, one cannot expect to produce an infinite periodic Chern-Simons potential. This is most easily understood by the definition of the Chern-Simons number in the $2 \mathrm{~d}$ abelian model:

$$
n_{c s}\left(n_{\tau}\right)=\frac{1}{2 \pi} \sum_{n=1}^{N_{\sigma}} \theta_{1}\left(n, n_{\tau}\right)
$$

Since $\theta_{1} \in[-\pi ; \pi]$ the possible Chern-Simons numbers lie in the interval $[-N / 2 ; N / 2]$. By dividing through the measure a periodic potential, which is restricted into some interval, is produced (fig. 3). ${ }^{14}$ There is also an indication for a more flat distribution at higher temperature indicating a higher transition rate.

In analogy to the $2 \mathrm{~d}$ abelian model the distributions will be corrected for the $4 \mathrm{~d}$ $S U(2)$ model. The same behaviour becomes transparent, thus for both models the periodic structure of the distribution after dividing out the measure will be assumed from now on. ${ }^{14}$ Therefore it suffices to shift all Chern-Simons numbers by integers into the intervall $[-1 / 2 ; 1 / 2]$ in order to investigate the form of the potential.

Investigating now the volume and temperature dependence of the distributions gives the following picture: The distributions get more broad with higher temperature, but there also is an increasing width with higher volume. While the temperature dependence will be investigated in detail, the volume dependence makes it impossible to investigate the temperature influence on large volumes. ${ }^{15}$

This is related to the presence of high Chern-Simons fluctuations even at small temperature. These zero temperature fluctuations are irrelevant for vacuum transitions and have to be taken into account.

One possibility might be to smoothen the configurations in a way similar to the cooling method. ${ }^{16}$ However we used a different way out: At higher gauge coupling $\beta$ 
$16 \times 16$ lattice

$16 \times 2$ lattice

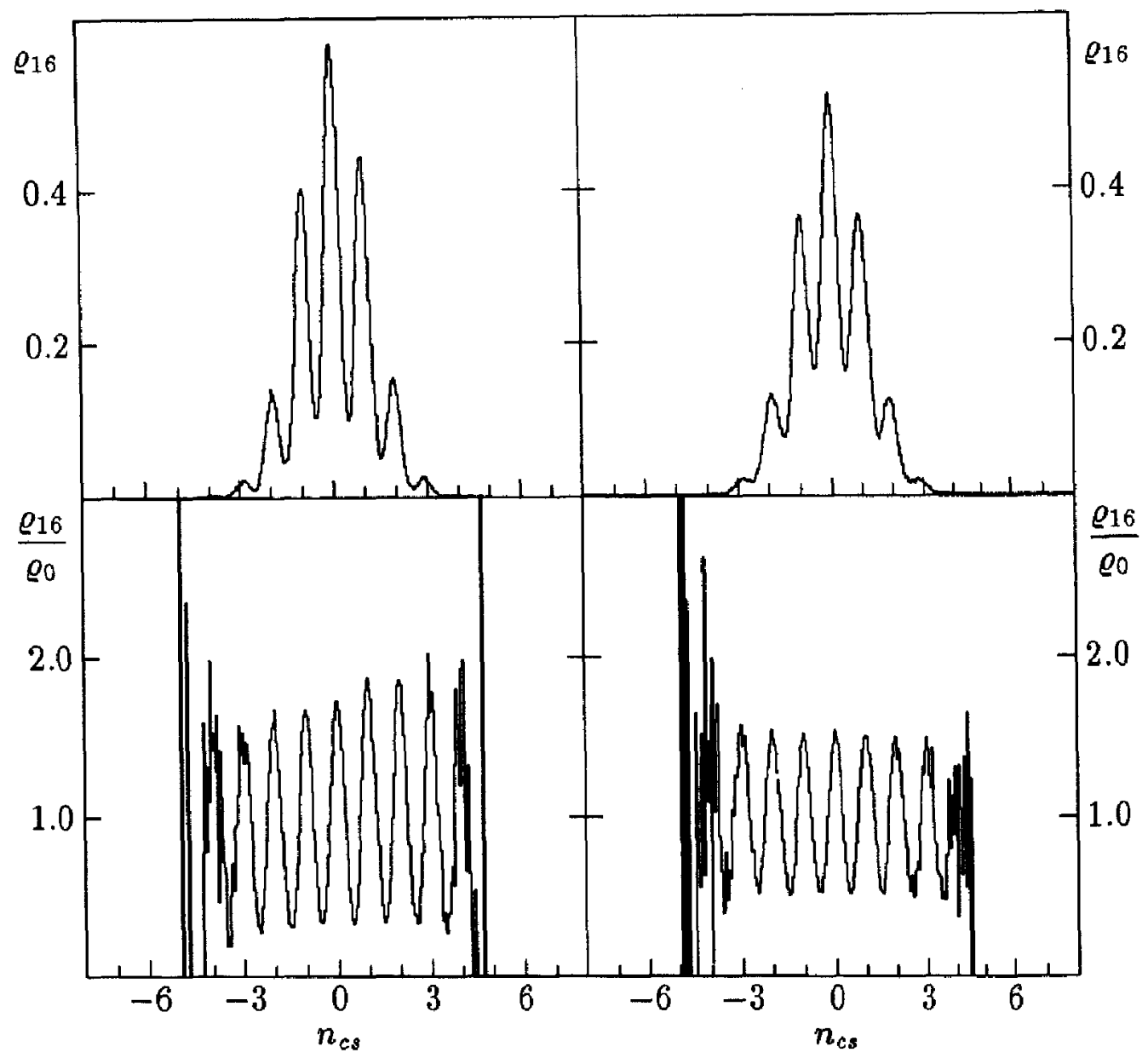

Fig. 3. Chern-Simons distributions (above) for zero (left) and finite temperature (right). Below the distributions are divided by the measure.

the field configurations become more smooth, resulting on average in smaller ChernSimons numbers. We chose the couplings $\beta=8, \kappa=0.12996$ and $\lambda=0.0017235$ corresponding to Higgs and vector boson masses of 0.5 in lattice units. ${ }^{17}$ Then the broadening of the distributions with rising temperature can be seen clearly (fig. 4).

Our data do not allow any conclusions concerning transition rates. Still it is possibel to draw some conclusions. Let us assume, that Chern-Simons numbers in the vicinity of half integer values indicate possible transitions. The increasing amount of half integer Chern-Simons numbers at higher temperature is then proportional to the increasing transition rate. Therefore the ratios of half valued Chern-Simons numbers at different temperature is compared with the corresponding ratios of transition rates.

These data indicate that at temperatures of two times the phase transition temperature there is still some exponential supression of the transitions (tab.2). It would be a confirmation of our picture if at higher temperature this supression would disappear. For such simulations a different approach would be needed, 


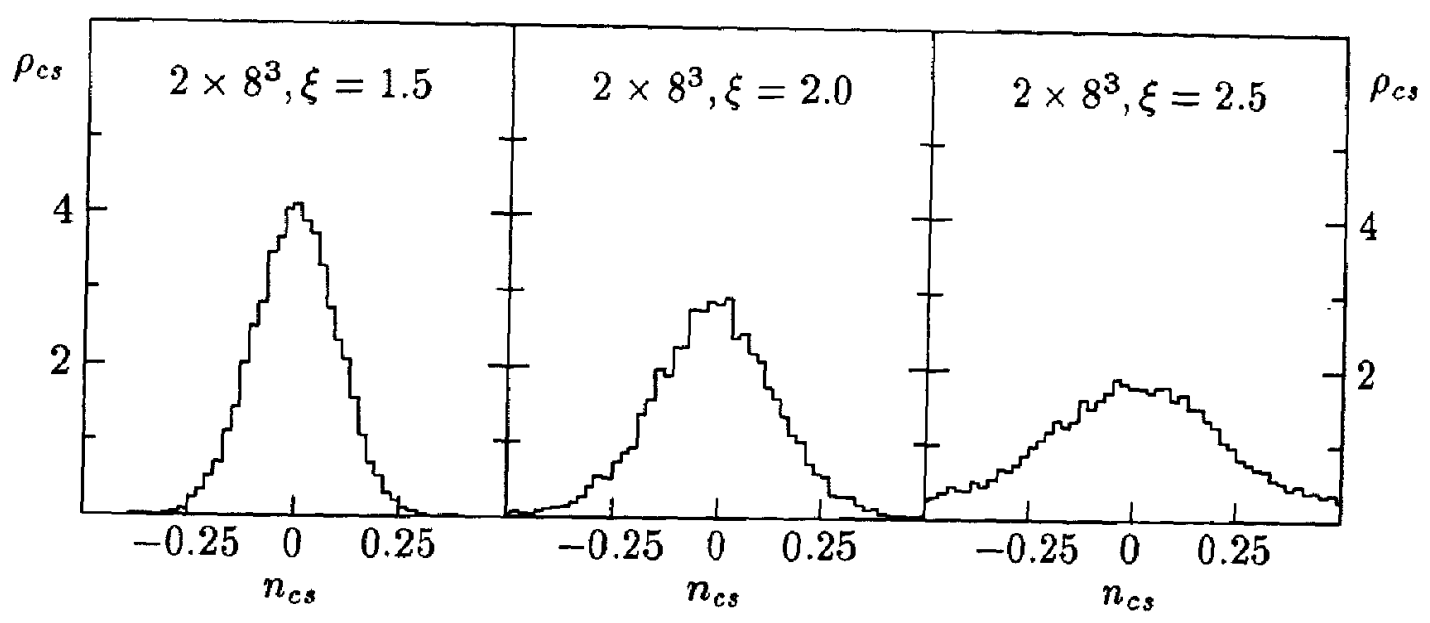

Fig. 4. Chern-Simons number distributions on $8^{3} \times 2$ lattice with anisotropies $\xi=1.5,2,2.5$. With increasing temperature the distributions get significantly broader.

Table 2. The first table gives the fraction Chern-Simons numbers, $F(\varepsilon, \xi)$, calculated on $2 \times 8^{3}$ lattices with anisotropy $\xi$ which differ from half-integer values by less than $\varepsilon$. In the second table we compare some ratios, $F\left(\varepsilon, \xi_{1}\right) / F\left(\varepsilon, \xi_{2}\right)$, with the semi-classical estimate for the tunneling rate in the two limiting cases $m_{W}=m_{W}(T=0)$ (eq. 3.3) (a) and $m_{W} \sim T$ (eq.3.4) (b), respectively.

\begin{tabular}{|c|c|c|}
\hline$\xi$ & $F(0.1, \xi)$ & $F(0.05, \xi)$ \\
\hline 1.8 & $0.0035(13)$ & $0.0013(07)$ \\
2.0 & $0.0109(23)$ & $0.0044(11)$ \\
2.5 & $0.0710(45)$ & $0.0327(28)$ \\
\hline
\end{tabular}

\begin{tabular}{|c|c|c|r|c|}
\hline$\xi_{1} / \xi_{2}$ & $\varepsilon=0.1$ & $\varepsilon=0.05$ & $(a)$ & $(b)$ \\
\hline $2.0 / 1.8$ & $3.1 \pm 1.3$ & $3.4 \pm 2.0$ & 3.9 & 1.5 \\
$2.5 / 2.0$ & $6.5 \pm 1.4$ & $7.4 \pm 2.0$ & 10.0 & 2.4 \\
\hline
\end{tabular}

since numerical difficulties arise at even higher lattice anisotropies. In this case it might be reasonable to work directly with the dimensionally reduced model in three dimensions. ${ }^{18}$

\section{Perspectives}

Our results indicate that it is possible to extend the low and high temperature estimates by lattice calculations. This is mainly due to the definition of the ChernSimons number on the lattice.

In the range of one up to 2.5 times the phase transition temperature the numerical calculations proved to be successfull. It would be desirable to simulate the system at even higher temperature, especially in order to check where the high temperature approximation becomes valid.

The 4 dimensional $S U(2)$ Higgs model is not suited for this purpose. To rise the temperature requires even higher values of $\xi$, which cannot be chosen arbitrary. Therefore it will probably be usefull to investigate the dimensionally reduced three 
dimensional effective model.

Future investigations should find faster methods to evaluate Chern-Simons numbers. There is also some requirement to take the zero temperature fluctuations into account. A method similar to cooling is desirable und might also speed up the numerical integrations due to its smoothening of the field configurations.

We conclude that numerical Monte Carlo simulations are important in order to investigate vacuum structure and transitions in Higgs models at finite temperature..

\section{References}

1. A. H.,Guth, Phys. Rev. D 23 (1981) 347.

2. P. Langacker, Recent aspects of GUT phenomenology, in: Ninth Workshop of Grand Unification, p. 3, ed.: R. Barloutaud.

3. G. 't Hooft, Phys. Rev. Lett. 37 (1976) 8; Phys. Rev. D 14 (1976) 3432; Erratum Phys. Rev. D 18 (1978) 2199.

4. D. V. Nanopoulos and S. Weinberg, Phys. Rev. D 20 (1979) 2484; A. Yu. Ignatiev, N. V. Krasnikov, V. A. Kuzmin and A. N. Tavkhelidze, Phys. Lett. B 76 (1978) 436, A. Yu. Ignatiev, V. A. Kuzmin and M. E. Shaposhnikov, Phys. Lett. B 87 (1979) 114.

5. M. Dine, R. G. Leigh, P. Huet, A. Linde, D. Linde, Phys. Rev. D 46 (1992) 550.

6. I. Bender, T. Hashimoto, F. Karsch, V. Linke, M. Schiestl, and I. O.Stamatescu, Nucl. Phys. B (Proc. Suppl.) 20 (1991) 329; M. Schiestl, Skalare Modella und Higgsmodelle auf Gittern mit anisotropen Kopplungen, PhD thesis, Heidelberg 1991.

7. E. Mottola and S. Raby, Phys. Rev. D 42 (1990) 4202.

8. R.F.Dashen, B. Hasslacher and A. Neveu, Phys. Rev. D 10 (1974) 4138; N. Manton, Phys. Rev. D 28 (1983) 2019; F. Klinkhammer and N. Manton, Phys. Rev. D 30 (1984) 2212.

9. P. Arnold and L. McLerran, Phys. Rev. D 36 (1987) 581, Phys. Rev. D 37 (1988) 1020.

10. J. Ambjørn, T. Askgaard, H. Porten and M. E. Shaposhnikov, Phys. Lett. B 244 (1990) 479; Nucl. Phys. B 353 (1991) 346;

11. N. Seiberg, Phys. Lett. B 148 (1984) 456.

12. H. G. Evertz, J. Jersák, C. B. Lang and T. Neuhaus, Phys, Lett. B 171 (1986) 271.

13. D. J. Gross, R. D. Pisarski and L. G. Yaffe, Rev. Mod. Phys. 53 (1981) 43.

14. F. Karsch, M. L. Laursen, T.Neuhaus, B. Plache and U.-J. Wiese, Int. J. Mod. Phys. C 3 (1992) 811; F. Karsch, M. L. Laursen, T. Neuhaus and B. Plache, Nucl. Phys. B (Proc. Suppl.) 30 (1993) 715.

15. F. Karsch, M.L.Laursen, T. Neuhaus and B. Plache, Vacuum Tunneling and Periodic Structure in Lattice Higgs models, Bielefeld Preprint BI-TP 93/04, to appear in Nucl. Phys. B; Thermal fluctuations of Chern-Simons numbers in the Lattice SU(2) Higgs model, Bielefeld Preprint BI-TP 93/21.

16. A. Duncan and R. D. Mawhinney, Phys. Lett. B 282 (1992) 423.

17. B. Bunk, E.-M. Ilgenfritz, J. Kripfganz and A. Schiller, Phys. Lett. B 284 (1992) 371; Lattice studies at zero and finite temperature in the $S U(2)$ Higgs model at small couplings, BI-TP $92 / 46$.

18. K. Kajantie, K. Rummukainen and M. Shaposhnikov, A Lattice Monte Carlo Study of the Hot Electroweak Phase Transition, CERN-TH 6901/93. 